\title{
Sistemática para avaliação multicriterial de investimentos no desenvolvimento de produtos
}

\author{
Alejandro Germán Frank ${ }^{a *}$, Diego Vinicius Souza ${ }^{\mathrm{b}}$, \\ Márcia Elisa Echevestec ${ }^{\mathrm{c}}$, José Luis Duarte Ribeiro ${ }^{\mathrm{d}}$ \\ a*agerfrank@producao.ufrgs.br, UFRGS, Brasil \\ bdviniciuss@uol.com.br, UFRGS, Brasil \\ cecheveste@producao.ufrgs.br, UFRGS, Brasil \\ dribeiro@producao.ufrgs.br, UFRGS, Brasil
}

\begin{abstract}
Resumo
Este artigo apresenta uma sistemática para avaliação multicriterial de investimentos associados ao desenvolvimento de produtos. A sistemática proposta se apoia nos critérios-chave a serem considerados na tomada de decisão. Ela é baseada na utilização dos métodos de avaliação econômica de investimentos VPL e payback, assim como nas ferramentas de desenvolvimento de produtos, QFD (Quality Function Deployment) e SWOT (Strenght-WeaknessesOportunities-Threats), utilizadas como suporte para a construção do método de avaliação multicriterial MAUT (Multiattribute Utility Theory). A sistemática proposta apresenta duas contribuições principais: i) a análise integrada de três critérios-chave para a decisão de investimentos em novos produtos: rentabilidade (considerada na avaliação econômica), qualidade (considerada no QFD) e estratégia (considerada no SWOT) e ii) uma modificação nas ponderações utilizadas na avaliação do MAUT, que reduz a subjetividade da atribuição de valores. A abordagem proposta foi avaliada em um caso aplicado e os resultados obtidos são discutidos.
\end{abstract}

Palavras-chave

QFD. SWOT. MAUT. Investimentos. Desenvolvimento de produtos.

\section{Introdução}

0 gerenciamento do portfólio de novos projetos de produto a serem desenvolvidos é considerado um dos aspectos-chave para o sucesso das empresas. Segundo Rozenfeld et al. (2006), as empresas devem decidir quais projetos de desenvolvimento priorizar, considerando as restrições de capital, tecnologia e competências. A avaliação das diferentes alternativas de investimento e a identificação dos projetos de produto prioritários permite aperfeiçoar a alocação dos recursos limitados, melhorando o desempenho global do desenvolvimento de produtos nas empresas. Assim sendo, fatores do desenvolvimento de produtos, tais como as estratégias de cada produto, o atendimento da qualidade demandada pelos clientes e a rentabilidade do projeto, devem ser avaliados na fase inicial do processo de desenvolvimento de produtos (PDP).

No entanto, muitas empresas realizam a análise da seleção das alternativas de investimento ponderando apenas alguns desses fatores. Usualmente, na escolha dos projetos de produto a serem desenvolvidos, priorizam-se principalmente os aspectos econômicos, sem considerar outros fatores qualitativos na decisão, como as estratégias corporativas e a qualidade demandada. Além disso, quando fatores qualitativos são considerados na gestão do portfólio, muitas vezes estes são avaliados sem o suporte de uma metodologia estruturada, que permita analisá-los e priorizá-los junto aos fatores econômicos dos projetos (SOUZA, 2008).

Porém, existem ferramentas aplicáveis ao desenvolvimento de produtos capazes de quantificar aspectos subjetivos. Dentre essas ferramentas, destacam-se o desdobramento da função qualidade (QFD - Quality Function Deployment), que possibilita desdobrar a qualidade demandada pelos clientes em características específicas dos produtos, e a análise de forças, fraquezas, oportunidades e ameaças (SWOT - Strength-Weaknesses-Opportunities-Threats), que permite analisar os fatores estratégicos envolvidos 
nos diferentes projetos de produtos. Além disso, características qualitativas e quantitativas podem ser avaliadas conjuntamente por meio dos métodos de avaliação multicriterial. Dentre eles, ressalta-se a teoria da utilidade multiatributo (MAUT - Multiattribute Utility Theory), por ser considerado um método de fácil implantação nas empresas (MIN, 1994; THEVENOT et al., 2006).

Assim sendo, o objetivo deste trabalho é apresentar uma sistemática para avaliação multicriterial de investimentos em projetos de novos produtos. Para isso, propõe-se utilizar de maneira integrada as ferramentas citadas anteriormente. Esta sistemática utiliza as ferramentas QFD e SWOT junto aos métodos de avaliação econômica valor presente líquido (VPL) e payback, como suportes para a avaliação multicriterial de investimentos, baseada na análise MAUT. Nessa sistemática propõe-se avaliar no portfólio de projetos de produtos: i) as características de qualidade das alternativas de investimento, por meio do QFD; ii) os aspectos estratégicos, com a análise SWOT; iii) a rentabilidade, com a análise do VPL; e iv) o tempo de recuperação do capital investido, através do payback. Finalmente, essas avaliações são integradas na análise MAUT para a obtenção de um ranking final das alternativas de investimento em produtos a serem desenvolvidos.

A apresentação do trabalho está dividida em três partes: i) discussão dos conceitos teóricos que fundamentam a sistemática proposta; ii) apresentação das etapas que compõem a sistemática; e iii) aplicação da sistemática em um caso prático que ilustra sua utilização.

\section{Análise SWOT}

A análise SWOT é uma ferramenta utilizada para o delineamento das estratégias, na qual se examina o relacionamento entre os fatores internos e externos que influenciam o negócio (MÜLLER, 2003). A ferramenta analisa os fatores internos: forças (Strenght) e fraquezas (Weaknesses), assim como os fatores do ambiente externo: oportunidades (Opportunities) e ameaças (Threats). A análise das relações entre esses fatores é realizada por meio da matriz SWOT (LEE; KO, 2000).

Na Tabela 1, pode-se visualizar a matriz SWOT composta por quatro quadrantes que definem as seguintes estratégias: i) desenvolvimento (relacionamento entre forças e oportunidades); ii) crescimento (entre forças e ameaças); iii) manutenção (entre fraquezas e oportunidades); e iv) sobrevivência (entre fraquezas e ameaças) (LEE; KO, 2000).

$\mathrm{Na}$ Tabela 1, pode-se observar que o relacionamento entre os fatores internos e externos é avaliado por meio de notas, que representam a importância estratégica de cada elemento. Nesse sentido, no quadrante de forças e oportunidades, atribuem-se as notas $r s o_{i j}$, que representam quanto uma força interna $S_{i}$ pode ser utilizada para aproveitar uma oportunidade externa $O_{i}$ No quadrante de forças e ameaças, calcula-se, mediante as notas $r s t_{i j}$ o grau em que uma força interna $S_{i}$ pode ser utilizada para reduzir uma ameaça externa $T_{j}$ No quadrante de fraquezas e oportunidades, avalia-se com as notas $r w o_{i j}$ quanto uma fraqueza interna $W_{i}$ pode ser reduzida pelas oportunidades $O_{j}$ existentes no mercado. E no quadrante fraquezas e ameaças, avalia-se com as notas $r w t_{i j}$ quanto uma fraqueza interna $W_{i}$ pode ser potencializada por uma ameaça externa $T_{j} \mathrm{O}$ somatório de todas as notas de cada quadrante determina os pesos finais: $l_{S O i j}, l_{S T i j}, l_{W O i j}$ e $l_{W T i j}$, referentes aos quadrantes de desenvolvimento, crescimento, manutenção e sobrevivência respectivamente. Esses pesos definirão qual estratégia é prioritária para a realidade da empresa (MÜLLER, 2003; LEE; KO, 2000).

Tabela 1. Matriz SWOT.

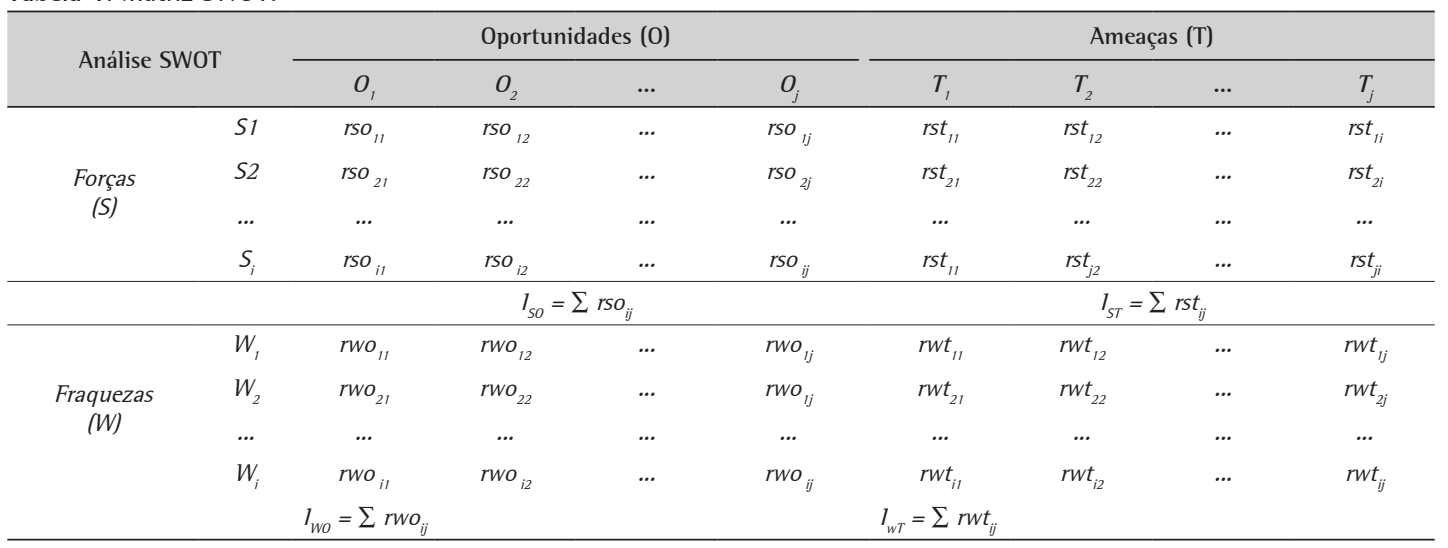


Nas etapas iniciais do desenvolvimento de produtos, após terem sido avaliados os fatores estratégicos utilizando a matriz SWOT, são definidas as características de qualidade que deveriam atender os possíveis produtos a serem desenvolvidos. Esta análise pode ser realizada por meio do QFD, que é apresentado a seguir.

\section{Matriz da qualidade do QFD}

O QFD é uma ferramenta que tem por objetivo transformar as necessidades dos clientes nos requisitos técnicos apropriados para o desenvolvimento de produtos. Essa ferramenta também pode ser considerada um guia para ser utilizado ao longo das fases de concepção e criação de novos produtos (GOVERS, 1996; CARNEVALLI; SASSI; MIGUEL, 2004; CARNEVALLI; MIGUEL, 2007). Na sua forma tradicional, o QFD é frequentemente apresentado contemplando quatro matrizes formadas por: i) matriz da qualidade; ii) matriz do produto (em referência a bens de consumo); iii) matriz de processos; e iv) matriz de recursos. Assim, o uso do QFD fornece o suporte para as equipes de desenvolvimento de produtos. Isto se deve ao fato de que essas quatro matrizes relacionam a demanda do consumidor com as especificações de engenharia, possibilitando o projeto dos componentes do produto e a definição das especificações das variáveis dos processos de produção (CHENG; MELO FILHO, 2007; AKAO, 1990; GOVERS, 1996).

Neste artigo será abordada apenas a matriz de qualidade (Figura 1), que enfatiza as características necessárias para o desenvolvimento conceitual do produto. Segundo Crawford e di Benedetto (2000), o desenvolvimento conceitual do produto é a etapa do PDP posterior à identificação das necessidades e oportunidades do produto, e consiste na criação de novas ideias para atender às necessidades dos clientes. Nesta etapa se utiliza a matriz da qualidade para traduzir as demandas identificadas junto ao

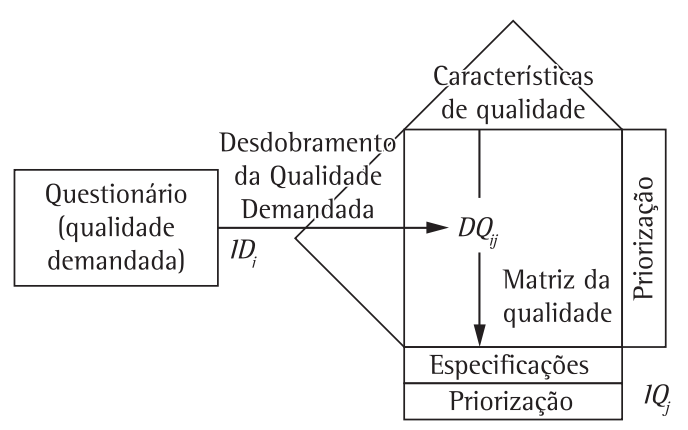

Figura 1. Exemplo de construção da matriz da qualidade do QFD. Fonte: adaptado de Ribeiro, Echeveste e Danilevicz (2001). cliente em requisitos do cliente e, posteriormente, em características de qualidade do produto ou requisitos do produto. (RIBEIRO; ECHEVESTE; DANILEVICZ, 2001; CHENG; MELO FlLHO, 2007). Contudo, no presente trabalho, utiliza-se o QFD já na avaliação do portfólio de produtos, antes da definição do projeto. Neste caso, constrói-se uma versão do QFD com informações preliminares sobre as características de qualidade que deveriam atender as alternativas de investimento que são avaliadas. Na medida em que o projeto é detalhado, essas características podem ser definidas com maior aprofundamento.

Para a construção do QFD, o ponto de partida é o levantamento da voz do cliente, que pode ser obtido por meio de uma pesquisa de mercado. A voz do cliente é inicialmente desdobrada, dando origem à qualidade demandada, que é uma das entradas da matriz da qualidade (RIBEIRO; ECHEVESTE; DANILEVICZ, 2001). Outra entrada dessa matriz são as características da qualidade, que são os requisitos técnicos do produto que podem vir a atender às necessidades do cliente. A seguir, preenche-se a relação entre cada um dos itens da qualidade demandada e das respectivas características da qualidade, como ilustra a Figura 1.

De posse dessas entradas, a matriz da qualidade tem como principal resultado uma priorização das características da qualidade $\left(1 Q_{\mathrm{j}}\right)$, que é o resultado da soma das notas $D Q_{i j}$ de cada coluna ponderadas pelo peso da qualidade demandada $I D_{i}$, como apresentado na Equação 1.

$l Q_{j}=\sum_{i=1}^{n} I D_{i} \times D Q_{i j}$

Os pesos $1 Q_{j}$ apontam as características de qualidade mais relevantes para atender às necessidades do cliente, que neste trabalho serão utilizadas posteriormente para a avaliação dos critérios no MAUT.

\section{Métodos de avaliação de investimento}

$\mathrm{Na}$ área da engenharia econômica existem diversos métodos que permitem avaliar a viabilidade dos investimentos no portfólio de projetos. No presente trabalho, esses métodos serão tratados de maneira sucinta, visto que são temas já discutidos na literatura específica. Estes temas aparecem em detalhe nos trabalhos de Casarotto Filho e Kopittke (2000). Os métodos que serão apresentados são: o valor presente líquido (VPL) e o período de recuperação do capital investido (payback), considerando-se esses métodos como alguns dos mais tradicionais da área.

0 VPL calcula a diferença entre o valor presente do fluxo de caixa dos $t$ períodos durante o projeto $\left(F C_{t}\right)$, confrontando as receitas menos as despesas 
dos períodos, e o investimento incial requerido $\left(\mathrm{FC}_{0}\right)$, conforme apresentado na Equação 2. Nessas diferenças, considera-se a taxa de interesse (taxa de mínima atratividade - TMA) da empresa, visto que avaliam-se lançamentos futuros do projeto. Nesse sentido, quando o VPL é maior que zero, significa que o mesmo supera as expectativas dos investidores em relação à TMA fixada por estes (GALESNE; FENSTERSEIFER; LAMB, 1999; COPELAND; WESTON; SHASTRI, 2005; SOUZA, 2008).

$$
V P L=\sum_{t=1}^{n} \frac{F C_{t}}{(1+T M A)^{t}}+F C_{0}
$$

Assim sendo, quando comparados os VPL de diferentes alternativas de investimento no mesmo período de tempo, avalia-se a rentabilidade dos mesmos. No entanto, alternativas mais rentáveis podem levar maior tempo para que se recupere o capital investido. Por conseguinte, utiliza-se o payback descontado para avaliar o tempo que os projetos demoram para se pagarem considerando a TMA da empresa (COPELAND; WESTON; SHASTRI, 2005). 0 payback descontado é calculado fazendo $\mathrm{VPL}=0$ na Equação 2. Assim, o payback corresponde ao período $t$ necessário para que o VPL seja zerado.

\section{Método de avaliação multicriterial - MAUT}

O MAUT é uma abordagem analítica usada para auxiliar a tomada de decisão envolvendo múltiplos atributos ou critérios, como os aspectos estratégicos, econômicos e de qualidade apresentados anteriormente (SOUZA, 2008). Essa abordagem permite escolher entre um número de alternativas baseadas nos critérios que são avaliados. Utilizando o MAUT, o tomador de decisões pode maximizar a função utilidade que depende desses critérios, determinando a melhor alternativa dentre todas as avaliadas (THEVENOT et al., 2006). A forma da função utilidade dependerá das condições de independência dos critérios, que são independência em utilidade e independência aditiva. Quando houver a independência aditiva entre dois atributos, a função utilidade pode adquirir a forma de uma função aditiva, que assume que não há interação entre os critérios. Maiores detalhes referentes à forma da função utilidade podem ser vistos em Miranda e Almeida (2004).

Segundo Souza (2008), o MAUT permite uma avaliação mais abrangente que outros métodos multicriteriais, possibilitando a comparação de inúmeras alternativas. Outra vantagem é a transparência do método, que é de fácil desenvolvimento e aplicação, pois envolve operações matemáticas mais simples que outros métodos. Min (1994) ressalta outros pontos positivos desse método, como a possibilidade de avaliar vários atributos conflitantes entre si e realizar análises de sensibilidade. Também existem versões simplificadas do MAUT, como o SMART e o SMARTER. O SMART é utilizado para simplificar o uso do método em casos nos quais os dados possuem grande subjetividade, utilizando escalas padronizadas na atribuição dos pesos de cada critério (KONIDARI; MAVRAKIS, 2007). Por outro lado, quando é difícil estabelecer as importâncias entre os critérios, podem ser utilizados dados ordinais, com os quais será mais fácil organizar as prioridades. Nesses casos, pode ser utilizado o SMARTER (SHEPETUKHA; OLSON, 2001). Ainda, cada pessoa pode avaliar um mesmo critério de diferentes maneiras, em função de sua atitude frente a riscos. Nesse caso podem ser utilizadas modelagens probabilísticas multicriteriais (MIRANDA; ALMEIDA, 2004).

No presente trabalho, é utilizado o MAUT, e a dificuldade de decidir a respeito da prioridade dos critérios e importância das alternativas é abordada através do uso de outros métodos. 0 presente trabalho adota métodos econômicos, VPL e payback, e métodos qualitativos, QFD e SWOT, para avaliar as prioridades das alternativas de investimento. Os resultados desses métodos são utilizados como inputs do MAUT, reduzindo assim a dificuldade de atribuição dos pesos dos critérios. 0 uso conjunto desses métodos ajuda a estruturar melhor a tomada de decisões no MAUT, como é apresentado na seção seguinte.

\section{Apresentação da sistemática proposta}

A sistemática proposta serve de apoio à tomada de decisões inerentes às etapas do desenvolvimento de produtos. Ela ajuda a decidir em quais dos projetos de produto é melhor investir, considerando um portfólio de projetos com diferentes características. Nesse cenário, avaliar somente o retorno econômico de um investimento pode levar a uma decisão errada, pois o projeto pode influenciar as estratégias corporativas e a qualidade demandada pelo cliente. Assim sendo, uma má decisão de investimento pode implicar em não atendimento de mercados potenciais, afetando a médio ou longo prazo as receitas e imagem da empresa.

Desta maneira, a sistemática proposta inclui os fatores estratégicos, por meio da matriz SWOT, a qualidade demandada, por meio do QFD, e os fatores econômicos, por meio do VPL e payback. Os resultados da aplicação desses métodos são utilizados como inputs para a avaliação multicriterial das alternativas de investimento, realizada no MAUT. Com essa integração, além de se estabelecer três critérios básicos a serem 


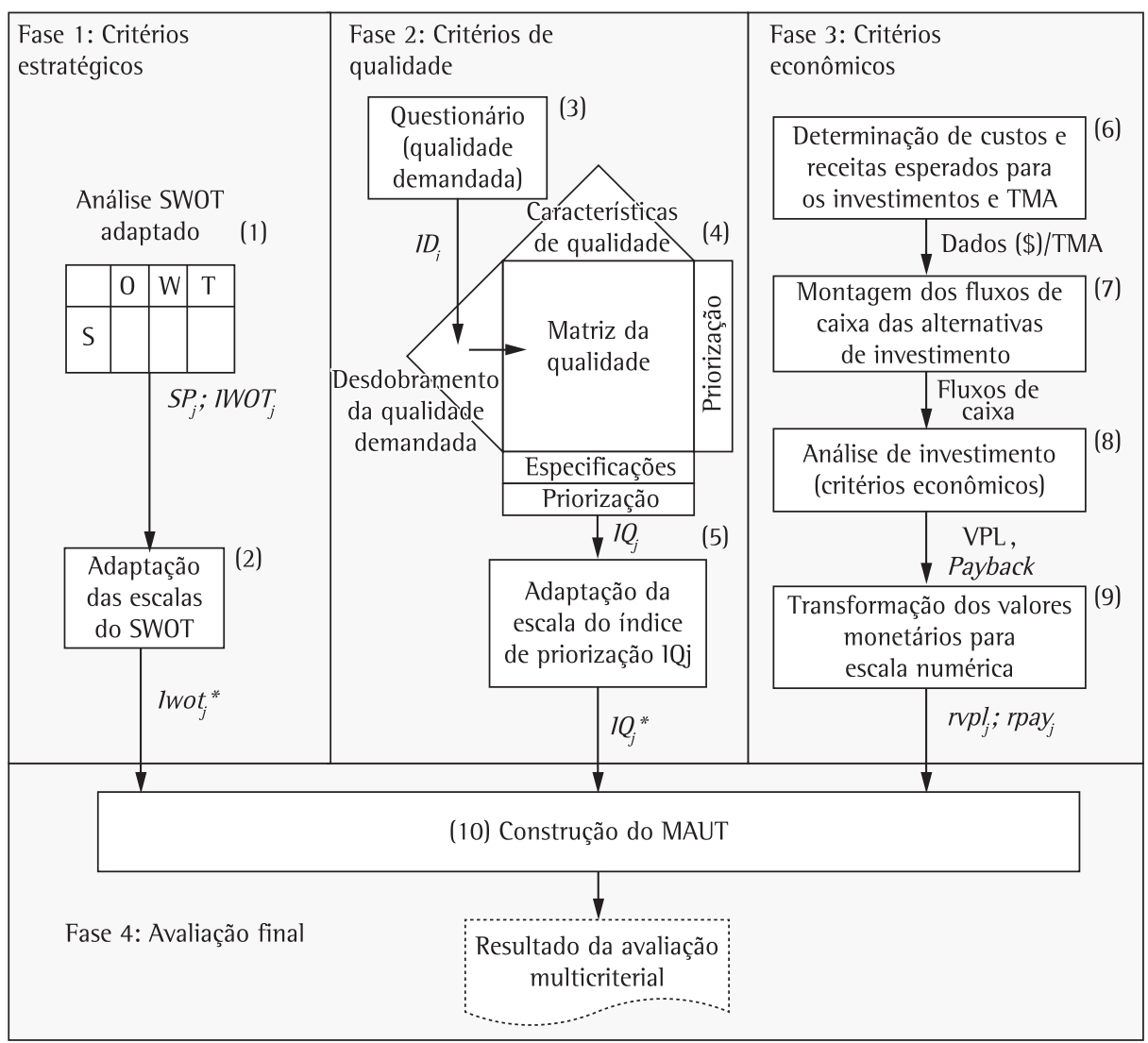

Figura 2. Sistemática para avaliação multicriterial de investimentos no desenvolvimento de produtos.

avaliados (estratégia, qualidade e rentabilidade), é possivel sistematizar a atribuição dos pesos usados na análise do MAUT, uma vez que a proposta utiliza métodos estruturados para obter as notas a serem preenchidas na matriz MAUT.

Para realizar a avaliação dos investimentos, segue-se uma sistemática composta por quatro fases principais, conforme apresentado na Figura 2: i) avaliação estratégica; ii) avaliação da qualidade; iii) avaliação da rentabilidade; e iv) priorização final das alternativas de investimento. Essas etapas são descritas a seguir.

\subsection{Fase 1: avaliação estratégica}

Inicialmente, procede-se à avaliação estratégica do negócio por meio da matriz SWOT. Esta fase permite que, posteriormente, as alternativas sejam comparadas, avaliando quais delas atendem melhor às características estratégicas do negócio.

A matriz SWOT usada na sistemática proposta apresenta algumas modificações em relação à versão tradicional. Nessa proposta, a melhor alternativa do ponto de vista estratégico será aquela que aproveite melhor as oportunidades existentes na empresa e ajude a reduzir tanto as fraquezas da empresa quanto as ameaças externas. Assim sendo, no SWOT adaptado realiza-se o cruzamento entre as forças da empresa e as fraquezas, ameaças e oportunidades do negócio, conforme apresentado na Tabela 2. Para a construção desta matriz, em primeiro lugar atribuem-se pesos para todas as forças $\left(I s_{i}\right)$, oportunidades $\left(l_{j}\right)$, fraquezas $\left(l w_{j}\right)$ e ameaças $\left(l t t_{j}\right)$ identificadas no negócio. Esses pesos definem o grau de influência que os fatores analisados têm sobre o negócio da empresa e são estabelecidos pela equipe de projetos. Os pesos atribuídos utilizam uma escala de 1 a 9 , seguindo a mesma lógica do preenchimento do QFD, onde 1 é uma importância muito baixa, e 9, uma importância muito alta.

Logo a seguir, realiza-se o preenchimento da matriz, adotando-se a mesma escala anteriormente explicada. No primeiro quadrante da matriz, avaliam-se os relacionamentos $r s_{i j}$, que representam quanto cada força $i$ da empresa pode contribuir para aproveitar a oportunidade $j$ identificada no mercado, sendo 1 um potencial muito baixo e 9 muito alto. As notas desse 
Tabela 2. Matriz SWOT adaptada.

\begin{tabular}{|c|c|c|c|c|c|c|c|c|c|c|c|c|c|}
\hline \multirow{2}{*}{\multicolumn{2}{|c|}{ Análise SWOT }} & \multicolumn{4}{|c|}{ Oportunidades $(0)$} & \multicolumn{4}{|c|}{ Fraquezas (W) } & \multicolumn{4}{|c|}{ Ameaças (T) } \\
\hline & & 10 & $\mathrm{Io}_{2}$ & ... & $1 o_{j}$ & $l w_{1}$ & $\mathrm{lw}_{2}$ & $\ldots$ & $l w_{j}$ & It & $1 t_{2}$ & $\ldots$ & $I t_{j}$ \\
\hline \multirow{4}{*}{ Forças (S) } & $l s s_{1}$ & $r s o_{t \prime}$ & $r s O_{t 2}$ & ... & $r s o_{l j}$ & $r s w_{n}$ & $r s w_{12}$ & $\ldots$ & $r s w_{l i}$ & $r s t_{n}$ & $r s t_{12}$ & $\ldots$ & $r s t_{1 i}$ \\
\hline & $l s_{2}$ & $r \mathrm{ro}_{21}$ & $r \mathrm{SO}_{22}$ & $\ldots$ & $r S O_{2 j}$ & $r s w_{21}$ & $r s w_{22}$ & $\ldots$ & $r s w_{2 i}$ & $l s t_{21}$ & $r s t_{22}$ & ... & $r s t_{2 i}$ \\
\hline & $\ldots$ & ... & $\ldots$ & ... & $\ldots$ & ... & ... & $\ldots$ & $\ldots$ & $\ldots$ & $\ldots$ & ... & $\ldots$ \\
\hline & $1 s_{i}$ & $r s O_{i t}$ & $\mathrm{rSO}_{\mathrm{i2}}$ & ... & $r s o_{i j}$ & $r s w_{n}$ & $r s w_{j 2}$ & $\ldots$ & $r s w_{j i}$ & $r s t_{n}$ & $r s t_{j 2}$ & $\ldots$ & $r s t_{j i}$ \\
\hline \multicolumn{14}{|c|}{$S P_{j}$} \\
\hline \multicolumn{14}{|c|}{ lwot $_{j}$} \\
\hline Iwo & & & & & & & & & & & & & \\
\hline
\end{tabular}

quadrante priorizarão projetos que possam aproveitar as novas oportunidades do negócio a partir das forças já existentes. No segundo quadrante, avaliam-se os relacionamentos $r s w_{i j}$, que representam quanto cada força $i$ da empresa pode ajudar a compensar a fraqueza $j$ existente. Nesse caso, a ausência de relacionamento é representada pela nota máxima da escala utilizada, pois no caso de não existirem forças que equilibrem as fraquezas os novos projetos deverão se focalizar nesse quadrante como prioritário. No terceiro quadrante, avaliam-se os relacionamentos $r s t_{i j}$ que representam quanto cada força $i$ ajuda a reduzir a ameaça $j$ do mercado. Esse preenchimento segue a mesma lógica do segundo quadrante de forças e fraquezas (Tabela 2).

Para a determinação dos pesos das oportunidades, ameaças ou fraquezas, primeiro calcula-se o índice $S P_{j}$ (Equação 3) como o somatório dos produtos entre as importâncias das $n$ forças identificadas ( $/ s_{j}$ ) e as notas dos preenchimentos da matriz $r_{i j}$ para a coluna $j$ avaliada (sendo $r_{i j}=r s O_{i j} ; r s w_{i j} ; r s t_{i j}$ ).

$$
S P_{j}=\sum_{1}^{n} l s_{i} \times r_{i j}
$$

Uma vez determinado o índice $S P$, os pesos das oportunidades, ameaças ou fraquezas ( $/$ wot $_{j}$ ) calculam-se então de acordo com a Equação 4.

$$
I w o t_{j}=\left(S P_{j}\right)^{(V A)} \times I p_{j}
$$

$\mathrm{Na}$ Equação 4, $I p_{j}$ representa o peso das oportunidades, ameaças e fraquezas $\left(I p_{j}=l o_{j} ; l w_{j} ; l t\right)$. Por outro lado, VA (calculado conforme a Equação 5) é um valor de ajuste da escala de $S P_{j}$ à escala do $I p_{j}$ que se utiliza para manter o equilíbrio entre as escalas dos dois índices.

$$
V A=\frac{\ln \left[\frac{\max _{j}\left(l p_{j}\right)}{\min _{j}\left(l p_{j}\right)}\right]}{\ln \left[\frac{\max _{j}\left(S p_{j}\right)}{\min _{j}\left(S p_{j}\right)}\right]}
$$

Finalmente, para a utilização dos pesos $l_{w o t}$ na matriz MAUT que será apresentada posteriormente, a escala deve ser ajustada para uma faixa de 0 a 1 , de maneira que seja compatível com as escalas das outras ferramentas que se integram na sistemática. 0 ajuste se realiza conforme a Equação 6 .

$$
\operatorname{Iwot}_{j}{ }^{*}=\frac{\operatorname{lwot}_{j}}{\max _{j}\left(\operatorname{lwot}_{j}\right)}
$$

\subsection{Fase 2: avaliação da qualidade}

Essa fase consiste na identificação das características de qualidade que devem atender aos projetos. Assim, uma vez estabelecidas essas características, pode-se avaliar quais alternativas têm maior potencial de atender às mesmas. A seguir descrevem-se as etapas dessa fase.

\subsubsection{Identificação da qualidade demandada}

Esta etapa consiste na elaboração de questionários para levantar os itens da qualidade demandada pelos clientes. Segundo Ribeiro, Echeveste e Danilevicz (2001), nessa etapa deve se realizar um questionário aberto ou estudos em grupos focados que permitam identificar aspectos gerais das preferências dos clientes. Na sequência, faz-se o desdobramento da qualidade demandada mediante uma estrutura hierárquica (árvore) que organiza e define as demandas de qualidade em uma tabela. Nesse trabalho, é utilizada uma versão simplificada desta tabela, pois a finalidade é diferente daquela associada à aplicação tradicional do QFD. Assim sendo, não se consideram a análise comparativa dos produtos concorrentes, o plano de melhoria e o argumento de vendas, que são aspectos normalmente analisados na matriz tradicional. Finalmente, considerando os pontos identificados, aplica-se um questionário fechado, onde é determinada a priorização da qualidade demandada $\left(\mathrm{ID}_{\mathrm{i}}\right)$ mediante a avaliação dos clientes de cada um dos itens utilizando uma escala de 1 a 10. 


\subsection{Elaboração da matriz QFD da qualidade demandada}

Os pesos da qualidade demandada (ID $)$, obtidos na etapa anterior, são utilizados na matriz da qualidade demandada. Nessa matriz, avaliam-se as relações entre cada uma das qualidades demandadas e as características de qualidade que quantificam essa demanda. Seguindo a metodologia de QFD apresentada por Ribeiro, Echeveste e Danilevicz (2001), para o preenchimento da matriz de qualidade realiza-se a seguinte pergunta: se a característica da qualidade $X$ for mantida em níveis excelentes, estará assegurado $\mathrm{o}$ atendimento da qualidade demandada Y? 0 grau com que a característica da qualidade assegura 0 atendimento da qualidade demandada é avaliado em uma escala de 0 a 9 , sendo 0 associado a nenhuma contribuição e 9 uma contribuição muito forte. Os pesos das características de qualidade (1Qj) são determinados mediante a soma ponderada das relações entre as características de qualidade e as demandas da qualidade, ponderando-se conforme os pesos das qualidades demandadas ( $\left(\mathrm{D}_{\mathrm{i}}\right)$ (Equação 1). A construção desta matriz segue o método tradicional do QFD, que pode ser encontrado em outros autores, como Ribeiro, Echeveste e Danilevicz (2001) e Cheng e Melo Filho (2007).

\subsection{Adaptação das escalas dos índices do QFD}

Os pesos $1 Q$, obtidos da matriz da qualidade, dependerão das priorizações da qualidade demandada obtidas no questionário fechado e da escala adotada nos cruzamentos nas matrizes de QFD. No questionário da qualidade demandada, usualmente, é adotada uma escala de 0\% a 100\%, para representar a importância relativa de cada aspecto demandado pelos clientes. Paralelamente, nas avaliações dos relacionamentos no interior das matrizes do QFD adota-se a escala 0 a 9. Desta maneira o peso $I Q_{j}$ terá uma escala que resulta do produto entre o peso $I D_{i}$ das priorizações obtidas no questionário e as avaliações dos relacionamentos da matriz QFD. Assim sendo, os valores $1 Q_{j}$ devem ser ajustados para seguir uma escala compatível com os outros índices que serão utilizados no MAUT. Para ajustar os valores $1 Q_{j}$, de maneira que sigam a escala de 0 a 1, utiliza-se a normalização segundo a Equação 7:

$$
I Q_{j}^{*}=\frac{1 Q_{\mathrm{j}}}{\max \left(1 \mathrm{Q}_{\mathrm{j}}\right)}
$$

\subsection{Fase 3: avaliação econômica}

Essa fase consiste na avaliação econômica das alternativas de investimento. Assim os resultados permitem comparar as alternativas e determinar quais os mais convenientes a partir dessa perspectiva. Os três primeiros passos dessa fase são: i) a determinação dos custos, receitas e a taxa mínima atratividade (TMA) para as duas alternativas de investimento; ii) a montagem dos fluxos de caixa; e iii) a análise dos fluxos de caixa por meio dos diferentes critérios econômicos escolhidos. Esses passos não serão aprofundados, visto que são temas amplamente discutidos na literatura específica da engenharia econômica (por exemplo, CASAROTTO FILHO; KOPITTKE, 2000).

Essas etapas consistem no levantamento dos dados sobre investimentos, receitas e despesas dos projetos, assim como definição da TMA para avaliar os projetos. A seguir se realiza a montagem dos fluxos de caixa, para finalmente avaliar os mesmos por meio dos métodos tradicionais do VPL e o payback.

\subsection{Transformação dos valores monetários para escala numérica}

Visto que a avaliação econômica das alternativas é em valores monetários, os mesmos devem ser transformados a notas em uma escala de 0 a 1 . Essa transformação foi feita utilizando uma curva $S$, que define limites máximos e mínimos fixos para a escala dentro dos quais a função adapta os valores monetários à mesma. Seguindo a função escolhida, as notas $r_{V P L j}$ do critério econômico baseado no VPL de cada alternativa $j$ são determinadas com a Equação 8.

$r_{V P L j}=1-e^{-\left(\frac{L+V P L_{j}}{\theta}\right)^{\gamma}}$

Na Equação 8, L é um parâmetro de localização que representa o máximo valor monetário de VPL que os especialistas estimam que poderia ser alcançado com o capital investido. Por outro lado, os parâmetros $\gamma$ e $\theta$ controlam respectivamente a forma e a amplitude da curva $S$, sendo estimados mediante as equações 9 e 10, onde $\mathrm{VPL}_{\max }$ é um valor considerado muito bom pelos especialistas, enquanto $\mathrm{VPL}_{\text {min }}$ é o um valor considerado muito ruim. Cabe ressaltar que o $\mathrm{VPL}_{\text {min }}$ pode adotar valores negativos em casos que o projeto não seja economicamente rentável, mas esteja sendo avaliado porque pode ajudar no posicionamento estratégico da empresa.

$$
\gamma=\frac{\ln \left(\frac{\ln (1-0,9)}{\ln (1-0,1)}\right)}{\ln \left(\frac{L+V P L_{\max }}{L+V P L_{\min }}\right)}
$$




$$
\theta=\frac{L+V P L_{\max }}{\left(-\ln \left(1-V P L_{\min }\right)\right)^{1 / \gamma}}
$$

Similarmente, o ajuste da escala do payback da alternativa $j$ é feito com a Equação 11, que utiliza uma curva $\mathrm{S}$ para transformar os valores para a escala 0 a 1 :

$r_{\text {Pay } j}=e^{-\left(\frac{L+\text { Paybackj }_{j}}{\theta}\right)_{\gamma}}$

Na Equação 11, $r_{\text {pay j }}$ é a nota para o payback da alternativa $j$. 0 parâmetro $L$ tem valor zero, considerando que a melhor condição do payback é o retorno imediato do capital investido $(L=0)$. Os parâmetros $\gamma$ e $\theta$ neste caso são estimados mediante as Equações 12 e 13 respectivamente, onde payback $_{\text {max }}$ é um valor considerado muito ruim pelos especialistas e payback ${ }_{\min }$ é um valor considerado muito bom para um projeto.

$$
\begin{gathered}
\gamma=\frac{\ln \left(\frac{\ln (0,1)}{\ln (0,9)}\right)}{\ln \left(\frac{\text { Payback }_{\text {max }}}{\text { Payback }_{\text {min }}}\right)} \\
\theta=\frac{\text { Payback }_{\text {min }}}{(-\ln (0,9))^{1 / \gamma}}
\end{gathered}
$$

Dessa maneira, obtêm-se as notas $r_{V P L j}$ e $r_{p a y j}$ para as alternativas avaliadas. Essas notas serão utilizadas posteriormente na construção da matriz MAUT.

\subsection{Avaliação final das alternativas de investimento}

Essa fase consiste na construção da matriz MAUT. Por meio desta, é possível realizar uma avaliação final das alternativas de investimento. Para alcançar esse intento, uma vez obtidos os pesos do SWOT, do QFD e da avaliação econômica, eles são utilizados como input para a construção do MAUT, conforme apresentado na Tabela 3. 0 primeiro passo da construção do MAUT é a determinação dos pesos dos seguintes critérios: estratégia, qualidade e econômico (pesos $I C Q, I C S$ e ICE respectivamente). Esses pesos devem ser estabelecidos pela empresa de acordo com suas prioridades, sendo a soma dos mesmos igual a 1 .

0 segundo passo do MAUT consiste na determinação dos pesos dos subcritérios. Para os elementos estratégicos, utilizam-se os pesos lwot $_{j}{ }^{*}$ obtidos na matriz SWOT. Para as características da qualidade são usados os pesos $l Q_{j}^{*}$ obtidos na matriz do QFD. Para os dois subcritérios econômicos, a empresa deve definir os pesos $I E$, que definem a prioridade entre os mesmos. Se for prioritária a boa rentabilidade do projeto, os resultados do VPL terão maior importância. Por outro lado, se for prioritário o retorno do investimento em menor tempo, o payback terá maior nota de importância relativa. Assim sendo, para esses dois subcritérios serão definidos os pesos $I E_{j}$ cuja soma é igual a 1.

Uma vez definidos os pesos dos subcritérios, os mesmos são normalizados considerando o peso global de cada critério (ICS, ICQ e ICE), conforme as

\begin{tabular}{|c|c|c|c|c|c|c|c|c|}
\hline \multirow{2}{*}{ Critérios } & \multirow{2}{*}{ Subcritérios } & \multirow{2}{*}{$\begin{array}{l}\text { Import. dos } \\
\text { critérios }\end{array}$} & \multirow{2}{*}{$\begin{array}{l}\text { Import. dos } \\
\text { subcrit. }\end{array}$} & \multirow{2}{*}{$\begin{array}{l}\text { Import. pond. } \\
\text { (IPn) }\end{array}$} & \multicolumn{4}{|c|}{ Notas comparativas entre os investimentos } \\
\hline & & & & & A1 & A2 & $\ldots$ & $\mathrm{A}_{k}$ \\
\hline \multirow{2}{*}{ Econômico } & VPL & \multirow{2}{*}{ ICE } & $I E_{1}$ & $I P n_{e l}$ & $r_{V P L I}$ & $r_{\text {VPL2 }}$ & $\ldots$ & $r_{V P L_{j}}$ \\
\hline & Payback & & $I E_{2}$ & $I p n_{e 2}$ & $r_{P A Y I}$ & $r_{\text {PAY } 2}$ & $\ldots$ & $r_{P A Y_{J}}$ \\
\hline \multirow{9}{*}{ Estratégia } & $O_{t}$ & \multirow{9}{*}{ ICS } & lwot, ${ }_{i}^{*}$ & $I P n_{s I}$ & $r s_{n}$ & $r s_{12}$ & $\ldots$ & $r s_{l j}$ \\
\hline & $\mathrm{O}_{2}$ & & lwot $_{i}^{*}$ & $I P n_{s 2}$ & $r s_{21}$ & $r s_{22}$ & $\ldots$ & $r s_{2 j}$ \\
\hline & $\ldots$ & & $\ldots$ & $\ldots$ & $\ldots$ & $\ldots$ & $\ldots$ & $\ldots$ \\
\hline & $w_{t}$ & & $\ldots$ & $\ldots$ & $\ldots$ & $\ldots$ & $\ldots$ & $\ldots$ \\
\hline & $W_{2}$ & & $\ldots$ & $\ldots$ & $\ldots$ & $\ldots$ & $\ldots$ & $\ldots$ \\
\hline & $\ldots$ & & $\ldots$ & $\ldots$ & $\ldots$ & $\ldots$ & $\ldots$ & $\ldots$ \\
\hline & $T_{1}$ & & $\ldots$ & $\ldots$ & $\ldots$ & $\ldots$ & $\ldots$ & $\ldots$ \\
\hline & $T_{\text {, }}$ & & $\ldots$ & ... & ... & $\ldots$ & $\ldots$ & $\ldots$ \\
\hline & $\ldots$ & & $\ldots$ & $\ldots$ & $\ldots$ & $\ldots$ & $\ldots$ & $\ldots$ \\
\hline \multirow{3}{*}{ Qualidade } & $Q_{1}$ & & $1 Q_{i}^{*}$ & $I P n_{q i}$ & $r q_{n}$ & $r q_{12}$ & $\ldots$ & $r q_{t j}$ \\
\hline & $Q_{2}$ & $I C Q$ & $1 Q_{2}^{*}$ & ... & $\ldots$ & $\ldots$ & $\ldots$ & $\ldots$ \\
\hline & $\ldots$ & & $\ldots$ & $\ldots$ & $\ldots$ & $\ldots$ & $\ldots$ & $\ldots$ \\
\hline \multicolumn{2}{|c|}{ Total } & 1 & -- & 1 & If & $l f_{2}$ & $\ldots$ & $I f_{j}$ \\
\hline
\end{tabular}
Equações 14, 15 e 16, onde $n$ representa: o número

Tabela 3. Construção do MAUT segundo a sistemática proposta. 
de elementos estratégicos (Equação 14); o número de características de qualidade (Equação 15); e o número de avaliações financeiras (Equação 16).

$$
\begin{aligned}
& I P_{n s i}=I C S \times \frac{I w o t_{j}^{*}}{\sum_{1}^{n} I w o t_{j}^{*}} \\
& I P_{n q i}=I C Q \times \frac{I Q_{j}^{*}}{\sum_{1}^{n} I Q_{j}^{*}} \\
& I P_{n e i}=I C E \times \frac{I E_{j}^{*}}{\sum_{1}^{n} I E_{j}^{*}}
\end{aligned}
$$

Finalmente, avaliam-se as notas comparativas entre as diferentes alternativas. Nesta etapa, já estão disponíveis as notas para os critérios econômicos $\left(r_{V P L j}\right.$ e $r_{P A Y_{j}}$ ), que foram avaliados nas etapas anteriores e que servem de input para esta parte da construção do MAUT. Para os critérios estratégicos e de qualidade precisa-se realizar ainda uma análise comparativa do desempenho de cada alternativa de investimento em relação ao critério avaliado. Assim sendo, realiza-se o preenchimento com as notas $r s_{i j}$ para os critérios estratégicos e as notas $r q_{i j}$ para os critérios de qualidade. Essa avaliação pode ser realizada de diferentes formas como em reuniões de engenharia ou utilizando questionários. A escala utilizada é de 0 a 1 , onde 0 representa que a alternativa analisada não contribui ou não atende o subcritério avaliado, enquanto 1 representa que a alternativa contribui intensamente ou atende muito bem ao subcritério avaliado.

A nota final $I f$ para a alternativa de investimento $j$ é calculada utilizando a Equação 17, onde $n$ representa: o número de elementos estratégicos e o número de características de qualidade respectivamente. Aquela alternativa que apresentar a maior nota será a mais viável considerando as três dimensões analisadas.

$$
\begin{aligned}
& I f_{j}=\sum_{i=1}^{n} I P n s_{i} \times r s_{i j}+\sum_{i=1}^{n} I P n q_{i} \times r q_{i j}+ \\
& +I P n e_{1} \times r_{V P L 1 j}+I P n e_{2} \times r_{P A Y 2 j}
\end{aligned}
$$

\section{Aplicação da proposta em um caso prático}

Nesta seção, é apresentada uma aplicação prática da sistemática de avaliação multicriterial proposta. A aplicação baseia-se em um problema real de uma empresa que fabrica móveis sob medida. Alguns dados foram alterados para manter o sigilo de informações consideradas confidenciais. Essas alterações, no entanto, não prejudicam a apresentação dos procedimentos propostos e a discussão dos resultados.
A avaliação multicriterial foi utilizada para subsidiar a decisão de investimentos para a terceirização do serviço de montagens dos móveis, que é considerado pela empresa como parte do próprio projeto de produto. Nesse tipo de produtos, o desenvolvimento está vinculado ao serviço da montagem final. Isto se deve ao fato de que a montagem influi sobre a qualidade final do produto, por ser um móvel sob medida, assim como sobre a qualidade do atendimento ao cliente, considerando que o fabricante deve visitar o cliente e montar o produto em sua casa. Dessa maneira, a empresa considera a montagem parte do projeto de produto que deve ser desenvolvido, dado que define as características finais dos móveis e influencia na imagem do produto.

No caso analisado, a empresa precisava decidir sobre os futuros investimentos para a ampliação da capacidade de desenvolvimento e fabricação de seus produtos. Nessa decisão se apresentavam três alternativas: i) A1: ampliar o serviço de montagens de móveis com investimentos próprios, ii) A2: manter o serviço atualmente disponível e terceirizar apenas a montagem nos novos mercados que serão atendidos, ou iii) A3: terceirizar todo o serviço de montagem dos móveis, concentrando-se somente no desenvolvimento e fabricação dos componentes do produto. Essa decisão, além de envolver custos econômicos diretos, também envolve a qualidade, pois o serviço de montagem tem uma relação direta com o cliente. Se o serviço for ruim, afeta diretamente a imagem da empresa, independentemente do serviço ser terceirizado. Portanto, a empresa teve que avaliar esses aspectos subjetivos, difíceis de quantificar, que influenciam diretamente nos futuros produtos a serem desenvolvidos. As avaliações apresentadas a seguir foram feitas pela equipe gerencial da empresa, contando com o suporte dos autores deste trabalho.

\subsection{Fase 1: avaliação estratégica}

A aplicação da sistemática começou com a avaliação dos critérios estratégicos. Em primeiro lugar se realizou a análise SWOT adaptada da empresa. No Quadro 1, apresentam-se os fatores estratégicos que foram confrontados na matriz SWOT da Tabela 4. No cálculo dos pesos $/$ wot $_{j}^{*}$, o valor de ajuste da escala de $S P_{j}$ à escala do $I p_{j}$ (Equação 5) foi de $V A=0,73$, visto que a proporção entre a escala dos pesos das oportunidades - ameaças - fraquezas e dos índices $S P_{j}$ é $3 / 4,5=0,73$. Com o uso do expoente VA, as duas escalas são equilibradas para que tenham igual influência no cálculo do $l w o t_{j}^{*}$. 
Quadro 1. Fatores estratégicos SWOT do negócio.

\begin{tabular}{|c|c|}
\hline \multirow{3}{*}{ Forças } & $\begin{array}{l}\text { 1- Focalização em um setor de clientes específico } \\
\text { que ainda não está sendo bem atendido. }\end{array}$ \\
\hline & $\begin{array}{l}\text { 2- Localização em zonas de importantes } \\
\text { investimentos imobiliários. }\end{array}$ \\
\hline & $\begin{array}{l}\text { 3- Infraestrutura da empresa está preparada para } \\
\text { atender às novas demandas de mercado. }\end{array}$ \\
\hline \multirow{3}{*}{ Fraquezas } & 1- Custos de produção elevados. \\
\hline & $\begin{array}{l}\text { 2- Falta de domínio técnico de materiais e } \\
\text { aplicações. }\end{array}$ \\
\hline & $\begin{array}{l}\text { 3- Ausência de uma estrutura formal de } \\
\text { desenvolvimento de novos produtos. }\end{array}$ \\
\hline \multirow{5}{*}{ Ameaças } & $\begin{array}{l}\text { 1- Possíveis mudanças na economia que } \\
\text { diminuam o investimento em imóveis. }\end{array}$ \\
\hline & 2- Possíveis novos concorrentes na região. \\
\hline & 3- Possivel escassez de alguns materiais \\
\hline & $\begin{array}{l}\text { 4- Dificuldades das empresas de transporte levar } \\
\text { o produto até os pontos de entrega. }\end{array}$ \\
\hline & $\begin{array}{l}\text { 5- Crescimento dos produtos que podem ser } \\
\text { substitutos. }\end{array}$ \\
\hline \multirow{3}{*}{ Oportunidades } & $\begin{array}{l}\text { 1- Incentivos fiscais para investimentos em novos } \\
\text { imóveis na região. }\end{array}$ \\
\hline & $\begin{array}{l}\text { 2- Novas tendências dos clientes para produtos } \\
\text { customizados. }\end{array}$ \\
\hline & $\begin{array}{l}\text { 3- Crescimento na região do setor fornecedor de } \\
\text { novos tipos de materiais. }\end{array}$ \\
\hline
\end{tabular}

\subsection{Fase 2: avaliação da qualidade}

A segunda etapa do trabalho consistiu na realização da matriz da qualidade, seguindo os preceitos do QFD. A aplicação iniciou com a identificação da qualidade demandada pelos clientes $\left(I D_{j}\right)$. Os aspectos demandados foram estabelecidos com base em pesquisas anteriores que a empresa já tinha realizado com os clientes. 0 passo seguinte foi traduzir a qualidade demandada $\left(I D_{j}\right) \mathrm{em}$ características de qualidade que permitem quantificar a demanda dos clientes $\left(1 Q_{j}\right)$. Essas características podem ser observadas na Tabela 5, onde é apresentada a matriz da qualidade.

\subsection{Fase 3: avaliação econômica}

Na terceira fase, foi realizada a avaliação econômica das alternativas de investimento. Neste caso foram considerados os seguintes pressupostos hipotéticos: i) a alternativa $\mathrm{A} 1$ terá um bom desempenho de VPL, mas intermediário no payback; ii) a alternativa A2 apresenta um VPL intermediário, mas é o melhor desde o ponto de vista do payback; e iii) a alternativa A3 apresenta o pior resultado em ambas as situações: VPL e payback. Contudo, observa-se nas fases anteriores que a

Tabela 4. Matriz SWOT do negócio.

\begin{tabular}{|c|c|c|c|c|c|c|c|c|c|c|c|c|c|}
\hline & & & \multicolumn{3}{|c|}{ Oportunidades } & \multicolumn{5}{|c|}{ Ameaças } & \multicolumn{3}{|c|}{ Fraquezas } \\
\hline & & & 1 & 2 & 3 & 1 & 2 & 3 & 4 & 5 & 1 & 2 & 3 \\
\hline \multicolumn{3}{|c|}{ Notas de importâncias } & 6 & 9 & 3 & 6 & 9 & 3 & 3 & 6 & 9 & 6 & 9 \\
\hline \multirow{3}{*}{ Forças } & 1 & 9 & 6 & 9 & 3 & 6 & 3 & 9 & 9 & 1 & 9 & 9 & 6 \\
\hline & 2 & 6 & 9 & 3 & 1 & 9 & 9 & 9 & 3 & 9 & 9 & 9 & 9 \\
\hline & 3 & 9 & 6 & 6 & 1 & 6 & 3 & 6 & 6 & 9 & 3 & 3 & 6 \\
\hline \multicolumn{3}{|c|}{$S P_{j}$} & 162 & 153 & 42 & 162 & 108 & 189 & 153 & 144 & 162 & 162 & 162 \\
\hline \multicolumn{3}{|c|}{ lwot } & 247 & 355 & 46 & 247 & 275 & 138 & 118 & 226 & 370 & 247 & 370 \\
\hline \multicolumn{3}{|c|}{$I w o t_{j}^{*}$} & 0,67 & 0,96 & 0,12 & 0,67 & 0,74 & 0,37 & 0,32 & 0,61 & 1,00 & 0,67 & 1,00 \\
\hline
\end{tabular}

Tabela 5. Matriz QFD da qualidade do caso analisado.

\begin{tabular}{|c|c|c|c|c|c|c|c|c|}
\hline & & 1 & 2 & 3 & 4 & 5 & 7 & \\
\hline & $\begin{array}{l}\text { Caracteristicas de qualidade } \\
\text { MATRIZ DA QUALIDADE } \\
\text { Qualidade demandada }\end{array}$ & 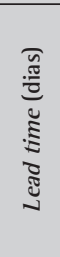 & 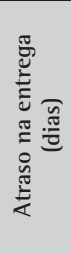 & 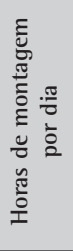 & 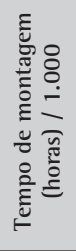 & 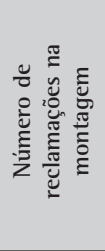 & 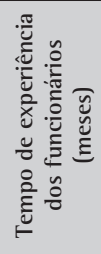 & $I D_{i}$ \\
\hline 1 & Entrega em prazo curto & 9 & 6 & 6 & 6 & 1 & 6 & 0,17 \\
\hline 2 & Entrega pontual & 6 & 9 & 3 & 3 & 1 & 6 & 0,15 \\
\hline 3 & Disponibilidade de horários adequados & 3 & 6 & 9 & 1 & 1 & 1 & 0,20 \\
\hline 4 & Rápida montagem & 3 & 6 & 6 & 9 & 1 & 9 & 0,14 \\
\hline 5 & Qualidade das terminações na montagem & 3 & 3 & 6 & 6 & 9 & 6 & 0,18 \\
\hline \multirow[t]{3}{*}{6} & Conhecimento do produto & 3 & 3 & 1 & 6 & 9 & 9 & 0,16 \\
\hline & $1 Q_{j}$ & 0,44 & 0,54 & 0,54 & 0,50 & 0,37 & 0,59 & \\
\hline & $1 Q_{j}^{*}$ & 0,75 & 0,92 & 0,91 & 0,84 & 0,64 & 1,00 & \\
\hline
\end{tabular}


alternativa A3 possui boas características em relação à estratégia e qualidade. Assim sendo, essa simulação permite analisar diferentes alternativas que poderiam se apresentar, considerando que as três alternativas têm alguma característica que pode torná-las atrativas, justificando-se a avaliação multicriterial.

Para a avaliação desta fase, em primeiro lugar definiram-se os parâmetros que permitiriam calcular posteriormente as notas dos resultados monetários dos fluxos de caixas. Assim sendo, definiram-se os seguintes parâmetros e restrições econômicas:

- VPL considerado muito bom $\left(V P L_{M a x}\right)=$ US\$50.000,00 (atribui-se nota 0,9 );

- VPL considerado muito ruim $\left(V P L_{m i n}\right)=-U S \$ 10.000,00$ (atribui-se nota 0,1 );

- Parâmetro $\mathrm{L}=$ US\$ 60.000,00 (considerado a condição máxima que poderia ser atingida);

- Parâmetro $\gamma=3,91$ (conforme a Equação 9);

- Parâmetro $\theta=88.870$ (conforme a Equação 10).

Com esses parâmetros obteve-se a proporção de escala, baseada na curva $S$ (Equação 8), conforme se apresenta na Figura 3. Os VPL para as três alternativas analisadas resultaram: $\mathrm{VPL}_{\mathrm{A} 1}=\mathrm{US} \$ 40.000,00$; $\mathrm{VPL}_{\mathrm{A} 2}=\mathrm{US} \$ 10.000,00$; e $\mathrm{VPL}_{\mathrm{A} 3}=\mathrm{US} \$ 9.000,00$. Utilizando-se 0 ajuste de escala, conforme a Equação 5, as notas para esses três VPL foram de $r_{\text {VPLA1 }}=0,80$; $r_{\mathrm{VPLA} 2}=0,25$ e $r_{\mathrm{VPLA} 3}=0,11$ respectivamente.

As restrições que foram definidas para o payback são as seguintes:

- Payback considerado muito bom $\left(\right.$ Pay $\left._{\min }\right)=1$ ano (para esta condição atribui-se nota 0,9$)$;

- Payback considerado muito ruim $\left(\right.$ Pay $\left._{\max }\right)=10$ anos (para esta condição atribui-se nota 0,1 );

- Parâmetro $\mathrm{L}=0$ anos (considerado a condição ideal que poderia ser atingida);

- Parâmetro $\gamma=1,34$ (conforme a Equação 12);

- Parâmetro $\theta=5,37$ (conforme a Equação 13).

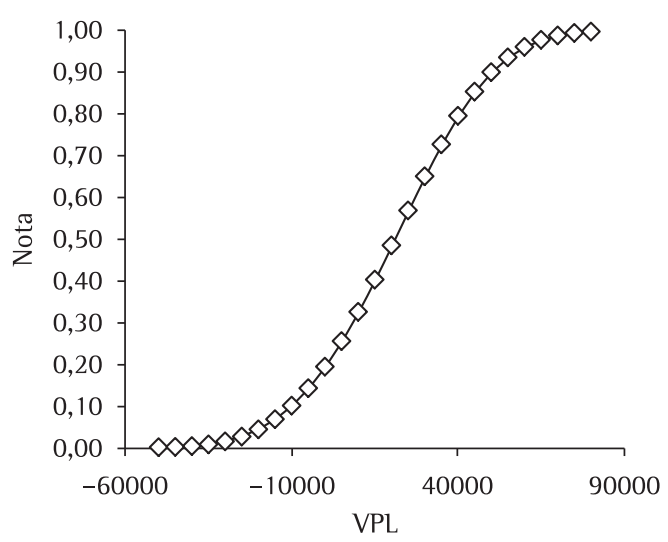

Figura 3. Transformação do VPL para a escala 0 a 1.
Com esses parâmetros obteve-se a proporção de escala para o payback, baseada na curva S (Equação 11), conforme se apresenta na Figura 4. Os payback para as três alternativas de investimento avaliadas foram: payback $_{A 1}=9$ anos; payback $_{A 2}=1$ ano e payback $k_{A 3}=15$ anos. Utilizando-se o ajuste de escala, conforme a Equação 8, as notas para esses três paybacks foram de $\mathrm{r}_{\mathrm{PAYA} 1}=0,14 ; \mathrm{r}_{\mathrm{PAYA} 2}=0,9$; $\mathrm{e}$ $\mathrm{r}_{\mathrm{PAYA} 3}=0,02$ respectivamente.

\subsection{Fase 4: avaliação final das alternativas de investimento}

Na última fase, o MAUT foi construído utilizando as notas obtidas nas fases anteriores, conforme apresentado na Tabela 6 . Antes de construir a matriz, foi analisado se os critérios utilizados são independentes (independência aditiva). Para ponderar os critérios, os gerentes da empresa atribuíram os seguintes pesos: $I C E=0,6 ; I C Q=0,25$ e $I C S=0,15$. Pode-se observar que a empresa valoriza mais os aspectos econômicos dos projetos, mas também considera os demais critérios com menor ponderação.

A seguir, foram incluídos na matriz os valores calculados para os pesos dos subcritérios. No caso dos aspectos estratégicos e de qualidade, os pesos já foram definidos nas fases anteriores. No entanto, para a avaliação econômica ainda é necessário definir se o VPL ou o payback é mais importante para a empresa. A empresa considerou que o VPL é mais importante $\left(\mathrm{IE}_{1}=0,7\right)$. Posteriormente são apresentadas algumas simulações dos resultados considerando uma importância artificialmente maior para o payback.

No preenchimento das notas comparativas entre as diferentes alternativas, para o critério econômico foram utilizadas as notas estabelecidas na fase 3 . Para os critérios de qualidade e estratégico foram avaliadas as três alternativas e preenchidas as notas,

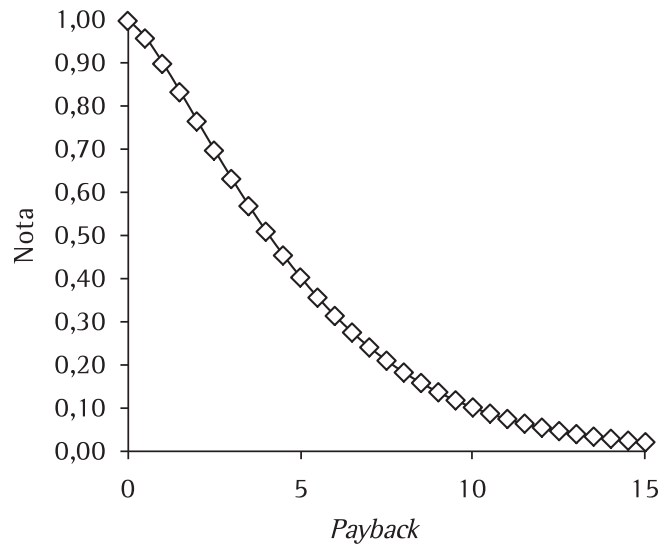

Figura 4. Transformação do payback para a escala 0 a 1. 
Tabela 6. Análise MAUT das alternativas de investimento.

\begin{tabular}{|c|c|c|c|c|c|c|c|c|}
\hline \multirow{2}{*}{ Critérios } & \multirow{2}{*}{ Subcritérios } & \multirow{2}{*}{$\begin{array}{c}\text { Pesos } \\
\text { dos } \\
\text { critérios }\end{array}$} & \multicolumn{3}{|c|}{ Pesos } & \multicolumn{3}{|c|}{ Alternativas } \\
\hline & & & Subcrit. & Norm. & Pond. & $\mathrm{A} 1$ & $\mathrm{~A} 2$ & A3 \\
\hline \multirow{2}{*}{ Econômico } & VPL & \multirow{2}{*}{0,60} & 0,70 & 0,70 & 0,420 & 0,80 & 0,25 & 0,11 \\
\hline & Payback & & 0,30 & 0,30 & 0,180 & 0,14 & 0,90 & 0,02 \\
\hline \multirow{11}{*}{ Estratégia } & $\begin{array}{l}\text { Incentivos fiscais para investimentos em novos imóveis } \\
\text { na região }\end{array}$ & \multirow{11}{*}{0,15} & 0,67 & 0,09 & 0,014 & 0,30 & 0,30 & 0,30 \\
\hline & Novas tendências dos clientes para produtos customizados & & 0,96 & 0,13 & 0,020 & 0,40 & 0,50 & 0,70 \\
\hline & $\begin{array}{l}\text { Crescimento na região do setor fornecedor de novos tipos } \\
\text { de materiais }\end{array}$ & & 0,12 & 0,02 & 0,003 & 0,60 & 0,60 & 0,80 \\
\hline & $\begin{array}{l}\text { Possíveis mudanças na economia que diminuam o invest. } \\
\text { em imóveis }\end{array}$ & & 0,67 & 0,09 & 0,014 & 0,30 & 0,30 & 0,30 \\
\hline & Possíveis novos concorrentes na região & & 0,74 & 0,10 & 0,016 & 0,50 & 0,60 & 0,70 \\
\hline & Possivel escassez de alguns materiais & & 0,37 & 0,05 & 0,008 & 0,60 & 0,70 & 0,80 \\
\hline & $\begin{array}{l}\text { Dificuldade das empresas. de transporte levarem o } \\
\text { produto até os pontos de entrega }\end{array}$ & & 0,32 & 0,04 & 0,007 & 0,30 & 0,30 & 0,40 \\
\hline & Crescimento dos produtos que podem ser substitutos & & 0,61 & 0,09 & 0,013 & 0,30 & 0,30 & 0,50 \\
\hline & Custos de produção elevados & & 1,00 & 0,14 & 0,021 & 0,40 & 0,50 & 0,70 \\
\hline & Falta de domínio técnico de materiais e aplicações & & 0,67 & 0,09 & 0,014 & 0,40 & 0,50 & 0,60 \\
\hline & $\begin{array}{l}\text { Ausência de uma estrutura formal de desenvolvimento de } \\
\text { novos produtos }\end{array}$ & & 1,00 & 0,14 & 0,021 & 0,50 & 0,50 & 0,60 \\
\hline \multirow{7}{*}{ Qualidade } & Lead time & \multirow{6}{*}{0,25} & 0,75 & 0,15 & 0,037 & 0,70 & 0,60 & 0,50 \\
\hline & Atraso na entrega & & 0,92 & 0,18 & 0,045 & 0,75 & 0,65 & 0,55 \\
\hline & Horas de montagem por dia & & 0,91 & 0,18 & 0,045 & 0,45 & 0,55 & 0,65 \\
\hline & Tempo de montagem & & 0,84 & 0,17 & 0,042 & 0,55 & 0,65 & 0,75 \\
\hline & Número de reclamações na montagem & & 0,64 & 0,13 & 0,031 & 0,35 & 0,45 & 0,75 \\
\hline & Tempo de experiência dos funcionários & & 1,00 & 0,20 & 0,049 & 0,55 & 0,55 & 0,70 \\
\hline & Total & 1,0 & & & 1,00 & 0,56 & 0,48 & 0,30 \\
\hline
\end{tabular}

Tabela 7. Simulação das possíveis alternativas.

\begin{tabular}{|c|c|c|c|c|c|c|}
\hline \multirow{3}{*}{ Avaliação } & \multirow{2}{*}{\multicolumn{3}{|c|}{$\begin{array}{c}\begin{array}{c}\text { Situação real: } \\
\text { maior importância } \\
\text { para VPL }\end{array} \\
\text { Alternativas }\end{array}$}} & \multirow{2}{*}{\multicolumn{3}{|c|}{$\begin{array}{c}\text { Situação simulada } \\
\text { maior importância } \\
\text { para payback }\end{array}$}} \\
\hline & & & & & & \\
\hline & $\mathrm{A} 1$ & $\mathrm{~A} 2$ & A3 & $\mathrm{A} 1$ & A2 & $\mathrm{A} 3$ \\
\hline Econômico & 0,60 & 0,45 & 0,08 & 0,33 & 0,71 & 0,05 \\
\hline Qualidade & 0,57 & 0,58 & 0,65 & 0,57 & 0,58 & 0,65 \\
\hline Estratégia & 0,41 & 0,47 & 0,60 & 0,41 & 0,47 & 0,60 \\
\hline Econômico - Qualidade & 0,59 & 0,49 & 0,27 & 0,41 & 0,67 & 0,24 \\
\hline Econômico - Estratégia & 0,55 & 0,45 & 0,22 & 0,36 & 0,64 & 0,20 \\
\hline Qualidade - Estratégia & 0,50 & 0,53 & 0,63 & 0,50 & 0,53 & 0,63 \\
\hline Todos os critérios & 0,56 & 0,48 & 0,30 & 0,40 & 0,64 & 0,28 \\
\hline
\end{tabular}

conforme apresentado na matriz da Tabela 6. Por fim, uma vez construído o MAUT, foram calculadas as notas finais para as três alternativas.

A Tabela 6 apresenta os resultados completos. Na Tabela 7, estão resultados obtidos a partir de diferentes combinações dos critérios, considerando cada um deles independentemente ou combinados. lsso permite visualizar como cada critério influencia na decisão final. Na Tabela 7 , observa-se que o investimento com melhor atratividade econômica foi priorizado, uma vez que foi atribuída uma nota de importância maior a essa dimensão. Também observa-se que, no caso do VPL ser priorizado (maior nota de importância), a alternativa A1 é a vencedora; entretanto, no caso de o payback ser priorizado, a alternativa A2 é a vencedora. Por outro lado, se forem considerados apenas os critérios estratégicos e de qualidade, a alternativa A3 seria a vencedora. Esse estudo de caso demonstra claramente a necessidade de incluir todos os critérios, devidamente ponderados, na avaliação dos investimentos.

\section{Considerações finais}

No presente trabalho, foi proposta uma sistemática de avaliação multicriterial de investimentos em projetos de desenvolvimento de produtos. Essa sistemática determina a prioridade entre as diferentes alternativas baseada no método MAUT, utilizando como suporte para elaboração do mesmo os métodos qualitativos QFD e SWOT, assim como os métodos de engenharia econômica VPL e payback.

A principal contribuição deste trabalho é, a partir da integração desses métodos, avaliar três critérios-chave dos projetos de produto: alinhamento estratégico, qualidade e rentabilidade. Isso é feito de forma sistematizada e mais objetiva que no uso tradicional do MAUT, onde os critérios são geralmente avaliados de maneira subjetiva por meio de discussões 
em equipe. A sistemática propõe passos sequenciais, que utilizam os métodos indicados para ajudar no processo de identificação dos critérios relevantes e determinação das ponderações dos mesmos.

A sistemática proposta envolve bom volume de coleta e tratamento dos dados. Em razão disso, acredita-se que é mais apropriada no estudo de investimentos que têm maior importância para a empresa, pois requer maior dedicação para as análises em comparação com a forma tradicional de aplicação do MAUT. Porém, os métodos apresentados são tradicionais na área de desenvolvimento de produtos e, assim sendo, acredita-se que o fato da sistemática possuir várias etapas não prejudica a sua aplicação prática.

Por fim, cabe ressaltar que o foco da sistemática proposta está dirigido para investimentos em projetos de produtos. Dessa maneira, um aspecto que pode ser considerado em futuras pesquisas é a aplicação dessa sistemática em outros tipos de investimentos.

\section{Referências}

AKA0, Y. Quality Function Deployment: integrating customer requirements into product design. Cambridge: Productivity Press, 1990.

CHENG, L. C.; MELO FILHO, L. D. R. QFD - Desdobramento da Função Qualidade na Gestão de Desenvolvimento de Produtos. São Paulo: Editora Blücher, 2007.

CARNEVALLI, J. A.; MIGUEL, P. A. C. Revisão, análise e classificação da literatura sobre o QFD - tipos de pesquisa, dificuldades de uso e benefícios do método. Gestão \& Produção, v. 14, n. 3, p. 557-579, 2007. http://dx.doi.org/10.1590/S0104530X2007000300011

CARNEVAlll, J. A.; SASSI, A. C.; MIGUEL, P. A. C. Aplicação do QFD no desenvolvimento de produtos: levantamento sobre seu uso e perspectivas para pesquisas futuras. Gestão \& Produção, v. 11 , n. 1, p. 33-49, 2004. http://dx.doi.org/10.1590/S0104530X2004000100004

CASAROTTO FILHO, N.; KOPITTKE, B. H. Análise de Investimentos: matemática financeira, engenharia econômica, tomada de decisão e estratégia empresarial. 9. ed. São Paulo: Atlas, 2000.

COPELAND, T. E.; WESTON, F. J.; SHASTRI, K. Financial Theory and Corporate Policy. 4th ed. Pearson Education, 2005.

CRAWFORD, C. M.; di BENEDETTO, C. A. New Products Management. 6th. ed. Boston: McGraw-Hill, 2000.

GALESNE, A.; FENSTERSEIFER, J. E.; LAMB, R. Decisões de Investimentos da Empresa. São Paulo: Atlas, 1999.
GOVERS, C. P. M. What and how about quality function deployment (QFD). International Journal of Production Economics, v. 4647, p. 575-585, 1996. http://dx.doi.org/10.1016/09255273(95)00113-1

KONIDARI, P.; MAVRAKIS, D. A multi-criteria evaluation method for climate change mitigation policy instruments. Energy Policy, v. 35, n. 35, p. 6235-6257, 2007. http://dx.doi.org/10.1016/j. enpol.2007.07.007

LEE, S. F.; KO, A. S. 0. Building balanced scorecard with SWOT analysis, and implementing "Sun Tzu's The Art of Business Management Strategies" on QFD methodology. Managerial Auditing Journal, v. 15, n. 1-2, p. 68-76, 2000. http://dx.doi. org/10.1108/02686900010304669

MIN, H. International Supplier Selection: A Multi-Attribute Utility Approach. International Journal of Physical Distribution \& Logistics Management, v. 24, n. 5, p. 24-33, 1994. http:// dx.doi.org/10.1108/09600039410064008

MIRANDA, C. M. G.; ALMEIDA, A. T. Visão multicritério da avaliação de programas de pós-graduação pela CAPES: o caso da área engenharia III baseado nos métodos ELECTRE 11 e MAUT. Gestão \& Produção, v. 11, n. 1, p. 51-64, 2004. http://dx.doi. org/10.1590/S0104-530X2004000100005

MÜLLER, C. J. Modelo de gestão integrando planejamento estratégico, sistemas de avaliação de desempenho e gerenciamento de processos (MEIO-Modelo de estratégia, Indicadores e Operações). 2003. Tese (Doutorado em Engenharia de Produção)-Universidade Federal do Rio Grande do Sul, Porto Alegre, 2003.

SHEPETUKHA, Y.; OLSON, D. L. Comparative Analysis of Multiattribute Techniques Based on Cardinal and Ordinal Inputs. Mathematical and Computer Modeling, v. 34, n. 1-2, p. 229-241, 2001. http:// dx.doi.org/10.1016/S0895-7177(01)00056-5

SOUZA, J. S. Proposta de uma sistemática para análise multicriterial de investimentos. 2008. Dissertação (Mestrado em Engenharia de Produção)-Universidade Federal do Rio Grande do Sul, Porto Alegre, 2008.

THEVENOT, H. J. et al. A multi-atribute utility theory-based approach to product line consolidation and selection. In: International Design Engineering Technical Conferences; Computers and Information in Engineering CONFERENCE, 2006, Philadelphia. Procedings... Philadelphia: ASME, 2006.

RIBEIRO, J. L. D.; ECHEVESTE. M. E.; DANILEVICZ, A. M. QFD - Desdobramento da Função Qualidade. Porto Alegre: Universidade Federal do Rio Grande do Sul, 2001. (Série Monográfica).

ROZENFELD, H. et al. Gestão de desenvolvimento de produtos: uma referência para a melhoria do processo. São Paulo: Saraiva, 2006.

\section{Agradecimentos}

Os autores agradecem à CAPES e ao CNPq pela concessão de bolsas de pesquisa e apoio financeiro.

\title{
A systematic analysis for multi-criteria evaluation of product development investments
}

\begin{abstract}
This paper presents a systematic analysis for multi-criteria evaluation of product development investments. The proposed systematic analysis is supported by key criteria to be considered during decision making. It is based on the use of traditional investment evaluation methods, such as NPV and payback, as well as in product development tools - QFD and SWOT. These tools are employed to assist the construction of the multi-criteria evaluation method, which follows the precepts of the Multi-attribute Utility Theory (MAUT). The proposed systematic analysis provides two main contributions: i) it integrates three key criteria concerning new product investment decisions: profit (considered by the economic evaluation), quality (considered by QFD), and strategy (considered by SWOT); ii) it includes a modification in the definition of the weights used by the MAUT evaluation, reducing the subjectivity of value attribution. The proposed systematic analysis was tested in an applied study and the results were discussed.
\end{abstract}

Keywords

QFD. SWOT. MAUT. Investments. Product development. 